\title{
Conceitos atuais sobre o crescimento e desenvol- vimento transversal dos maxilares e oportunidade de expansão mandibular
}

\author{
Adriano Marotta ARAUJO*, Peter H. BUSCHANG**
}

\begin{abstract}
Resumo
O interesse do ortodontista pelo crescimento e desenvolvimento do complexo crâniofacial vem se estendendo por décadas e inúmeros trabalhos já foram realizados nessa área. Necessitamos da compreensão do processo normal de maturação óssea e estruturas adjacentes para podermos diagnosticar uma deformidade dentofacial, bem como identificar fatores etiológicos intrínsecos e extrínsecos que atuam em conjunto, proporcionando alterações na dimensão transversal das bases ósseas e dos arcos dentários. Várias são as filosofias de tratamento para correção das deformidades transversais e recentemente com o advento de novos protocolos de tratamento, essa necessidade de compreensão do desenvolvimento das estruturas faciais parece ter aumentado. Restringindo-se às modificações transversais da maxila e da mandíbula do nascimento até a idade adulta, paradigmas foram instituídos e alguns perpetuam até hoje, entre eles a possibilidade ou não de expansão do arco dentário mandibular. Os propósitos desse trabalho são: 1) revisar alguns dos conceitos relacionados ao crescimento e desenvolvimento transversal dos maxilares presentes na literatura e 2) discutir a possibilidade de expansão no arco dentário mandibular.
\end{abstract}

Palavras-chave: Crescimento craniofacial. Expansão maxilar. Expansão mandibular.

\section{INTRODUÇÃO}

O famoso artista francês Meissonier disse uma vez "Nós somente vemos o que nós sabemos". Essa frase talvez nos ajude a entender um pouco mais da evolução dos estudos ortodônticos no sentido transversal. A discrepância transversal pode gerar más oclusões como a mordida cruzada posterior, o apinhamento dentário, constrição e assimetria dos arcos dentários. Quando isso ocorre o diagnóstico se torna mais rápido e preciso, mas normalmente uma discrepância no sentido transversal também está associada às alterações nos sentidos vertical e ântero-posterior. Essa associação, apesar de constante, não é uma regra, pois vários são os casos onde pacientes portadores da má oclusão Classe I, satisfatório equilíbrio vertical e ausência dos indicadores característicos da atresia apresentam arcos dentários transversalmente diminuídos. A literatura nos mostra que grande parte das pesquisas no ramo ortodôntico priorizava as discrepâncias nos sentidos ântero-posterior e vertical. Um dos responsáveis pelo aumento das pesquisas das dimensões transversais foi Dr. A. Haas, na década

Doutorado em Ortodontia - UNESP Araraquara / Baylor College of Dentistry, Dallas, Tx / Prof. do Curso de Graduação UNESP São José dos Campos / Prof. Titular do Curso de Graduação da UNISA - SP.

** Ph.D em Antropologia Física pela Universidade do Texas e Diretor Científico do Departamento de Ortodontia da Baylor College of Dentistry - Dallas, Tx. 
de 60, com a reintrodução da expansão ortopédica da maxila; assim, inúmeras investigações foram realizadas com o propósito de aumentar o conhecimento sobre essa terapia. Pesquisas também surgiram em pacientes sem tratamento, principalmente no campo de crescimento e desenvolvimento crâniofacial onde se julgou necessário entender o que era o normal e o correto, para se entender até que ponto a terapia com aparelhos está ou não interferindo e corrigindo uma má oclusão. A dimensão transversal passou a ser considerada também por outras filosofias de tratamento como a ortopedia funcional e a cirurgia ortognática.

A possibilidade de expansão da maxila já foi confirmada e considerada estável, desde que um período de contenção após a fase ativa seja preconizado. Com relação à expansão mandibular um paradigma foi instituído no início do século e ficou conhecido como a "Escola da Base Apical". Este paradigma foi baseado nos trabalhos de Axel Lundstrom $^{9}$, que determinou a base apical como o fator mais importante na correção da má oclusão. McCauley ${ }^{9}$ definiu que a distância intercanino e a distância intermolar deviam ser mantidas e nunca alteradas com o objetivo de aumentar a estabilidade do tratamento e, posteriormente, Strang e Nance $^{9}$ também confirmaram este postulado. Recentemente os trabalhos de Little e Herberger sugerem que a distância intercanino mandibular deve ser inalterável $^{11,16,17}$. Esses autores defendem a teoria de que a dimensão transversal do arco dentário mandibular não deve ser alterada durante o tratamento ortodôntico. Também encontramos na literatura trabalhos mostrando que a alteração da dimensão do arco dentário inferior é possível e relativamente estável ${ }^{6,12,15}$. Incertezas como esta nos despertaram o interesse na execução de uma revisão da literatura sobre o assunto e procuramos respostas para algumas questões que podem influenciar diretamente a conduta e estabilidade da terapia ortodôntica. Perguntas como: Quais são os centros de crescimento transversal da maxila e da mandíbula? Após a fusão da sutura sinfiseana, a mandíbula continua a crescer transversalmente? Podemos ou não alterar a distância intercanino? E a distância intermolar?

Portanto, o propósito desse trabalho foi revisar alguns dos conceitos relacionados ao crescimento e desenvolvimento transversal dos maxilares presentes na literatura e discutir a possibilidade de expansão no arco dentário mandibular.

\section{DESENVOLVIMENTO TRANSVERSAL DOS ARCOS DENTÁRIOS}

Inicialmente, iremos focalizar o desenvolvimento transversal dos arcos dentários e, numa segunda fase as alterações inerentes às bases ósseas. Variáveis como distância intercanino, distância intermolar, comprimento dos arcos dentários, entre outras, serão discutidas. Os trabalhos a seguir foram realizados em pacientes que nunca foram submetidos ao tratamento ortodôntico.

\section{ARCO DENTÁRIO SUPERIOR}

A necessidade de um banco de dados com valores normais do desenvolvimento oclusal nos seres humanos despertou o interesse de Moyers et al. ${ }^{21}$, que em 1976 publicaram um Atlas com valores normais da oclusão dentária. No total foram observados 208 indivíduos, 109 do gênero masculino e 99 do gênero feminino. Todas as mensurações transversais foram obtidas da menor distância entre os centróides dos dentes (ponto geométrico central do dente). A distância intercanino maxilar aumentou em média 1,25 $\mathrm{mm}$ dos 3 aos 7 anos de idade e 3,53 $\mathrm{mm}$ dos 7 aos 13 anos e praticamente se manteve até os 18 anos de idade. Moorrees et al..$^{20}$ observaram uma correlação entre o desenvolvimento transversal dos arcos dentários e a erupção dentária em uma amostra de 184 pacientes observada dos 3 aos 18 anos de idade. A distância intercanino aumentou progressivamente durante a dentição mista tanto para maxila como também para a mandíbula. As maiores alterações ( $3 \mathrm{~mm}$ ) ocorreram no período de transição dos incisivos decíduos para os incisivos permanentes para ambos os maxilares de ambos os gêneros (Fig. 1). Após essa fase, um segundo aumento foi observado 
Distância Intercanino Maxilar

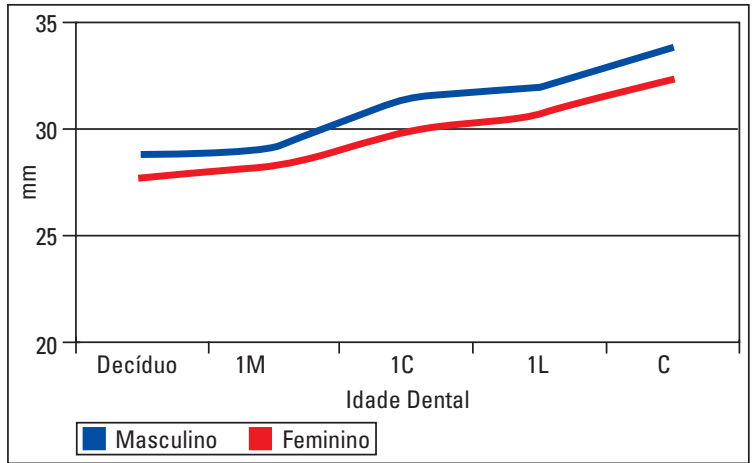

FIGURA 1 - Aumento da distância intercanino maxilar com relação à idade dental (irrupção dos dentes). Observe que o maior aumento ocorreu durante a troca dos incisivos central e lateral permanente. (MOORREES et al. ${ }^{20}, 1964$ ) $1 \mathrm{M}=$ primeiro molar permanente / IC= Incisivo central / IL=Incisivo lateral / $\mathrm{C}=$ Canino.

(1,5mm) depois da irrupção dos caninos permanentes, porém esse aumento só foi observado na maxila. Com relação ao comprimento do arco dentário durante a dentição mista foi observado uma diminuição para ambos os arcos da irrupção do primeiro molar até a irrupção do segundo molar permanente. A idade dental pareceu ser um parâmetro mais confiável do que a idade cronológica quando se diz respeito ao desenvolvimento dos arcos dentários. Ainda Moorrees et al. ${ }^{19}$ observaram em outro trabalho algumas alterações no comprimento do arco dentário. A primeira delas foi observada após a erupção dos primeiros molares permanentes com conseqüente fechamento dos possiveis diastemas existentes na dentição decídua. O mesmo ocorreu após a erupção dos incisivos permanentes, com maior intensidade para maxila do que para mandíbula. Outro fator que levou à diminuição do comprimento do arco foi o movimento mesial dos molares decíduos, especialmente os segundos molares. A forma do arco também alterou aumentando levemente na maxila e a migração mesial dos dentes durante o desenvolvimento dos arcos dentários foi caracterizado como um fenômeno fisiológico.

Björk e Skieller ${ }^{4}$ observaram que o aumento transversal do arco dentário superior está associado ao crescimento da sutura mediana. Para a distância intercanino o aumento entre 4 anos até a idade adulta foi em média de $3,1 \mathrm{~mm}$. Após a erupção dos primeiros molares o aumento foi de $1,1 \mathrm{~mm}$ até a idade adulta. Os autores ainda concluem que o arco dentário superior "escorrega" para frente com uma diminuição do comprimento do arco dentário e redução do espaço para os incisivos podendo isto levar a um possivel apinhamento secundário.

Athanasiou et al. ${ }^{1}$ revelaram que todas as estruturas esqueléticas da face aumentaram no sentido transversal dos 6 aos 15 anos de idade. Entretanto, a distância intermolar da maxila durante o período de 9 aos 12 anos não apresentou nenhum aumento. Sinclair e Little ${ }^{25}$ acompanharam o desenvolvimento do arco dentário da dentadura mista até a permanente. Sessenta e cinco pacientes participaram da amostra, sendo 33 do gênero masculino e 32 do gênero feminino. Os pacientes foram acompanhados em média dos 9 aos 20 anos de idade. Foi observado uma diminuição no comprimento do arco e da distância intercanino, mínima alteração da distância intermolar e aumento no índice de regularidade dos caninos. Durante todo o período a distância intercanino diminuiu $0,75 \mathrm{~mm}$ e a distância intermolar diminuiu $0,15 \mathrm{~mm}$. Bishara et al. ${ }^{2}$ estudaram as alterações transversais dos arcos dentários de 6 semanas até 45 anos de idade. Os resultados da primeira fase (até 2 anos de idade) mostraram um crescimento uniforme e significante nas regiões anterior e posterior de ambos maxilares para ambos os gêneros. $\mathrm{O}$ crescimento se manteve até 13 anos de idade. Após a completa erupção da dentição permanente houve uma diminuição na largura dos arcos dentários, especialmente na distância intercanino. A distância intercanino após 8 anos de idade praticamente permaneceu estável. Após essa fase as dimensões se mantiveram ou apresentaram ligeira redução até a idade adulta.

Para a idade adulta a literatura apresenta alguns estudos avaliando as modificações existentes após os 20 anos de idade. Bishara et al. ${ }^{3}$ concluíram que os arcos dentários se mantiveram estáveis ou mostraram pequenas alterações durante a fase adulta. Harris $^{10}$ estudou longitudinalmente as alterações no tamanho e na forma dos arcos dentários de 
Distância Intercanino dos 5 aos 17 anos

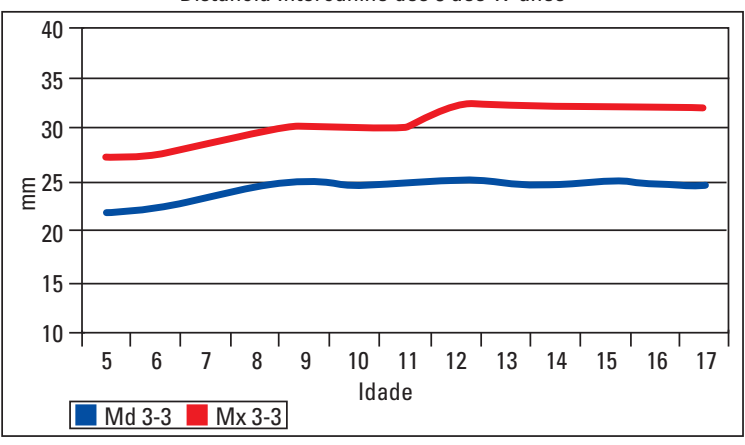

FIGURA 2 - Distância intercanino da maxila e da mandíbula dos 5 aos 17 anos de idade para o gênero masculino (MOYERS et al. ${ }^{21}, 1976$ ).

60 pacientes adultos. Os pacientes, 43 do gênero masculino e 17 do gênero feminino foram acompanhados em média por 35 anos. $\mathrm{O}$ primeiro exame ocorreu em média com 20 anos e o segundo com 55 anos. Havia pacientes com oclusão normal, má oclusão Classe I, II e III. Os resultados revelaram um aumento na dimensão transversal e diminuição no comprimento dos arcos dentários, sendo as alterações semelhantes para ambos os gêneros. A distância intercanino apresentou um aumento em média de $0,48 \mathrm{~mm}$ para a maxila e $0,14 \mathrm{~mm}$ para mandíbula. $\mathrm{O}$ mesmo ocorreu para a distância intermolar que apresentou um aumento de $2,41 \mathrm{~mm}$ e 2,29mm respectivamente para a maxila e mandíbula. $\mathrm{O}$ autor concluiu que durante o período avaliado os arcos se tornaram mais curtos e mais largos, e essas alterações não afetaram a coordenação entre eles, pois a sobremordida e sobressaliência se mantiveram. A origem desse aumento não foi bem definida, mas especulou-se a influência de forças oclusais sobre os arcos dentários com vetores de força mesial e vestibular na região posterior.

Bondevik ${ }^{5}$ estudou as alterações presentes na largura do arco dentário em pacientes observados dos 23 aos 35 anos de idade. Os modelos de estudo de 64 pacientes do gênero masculino e 80 pacientes do gênero feminino foram avaliados. As alterações observadas foram pequenas, nenhuma variável apresentou alteração superior a $0,55 \mathrm{~mm}$. A distância intercanino reduziu em ambos os arcos, sendo a maior alteração de $-0,18 \mathrm{~mm}$ para o gênero masculino. A distância intermolar aumen-

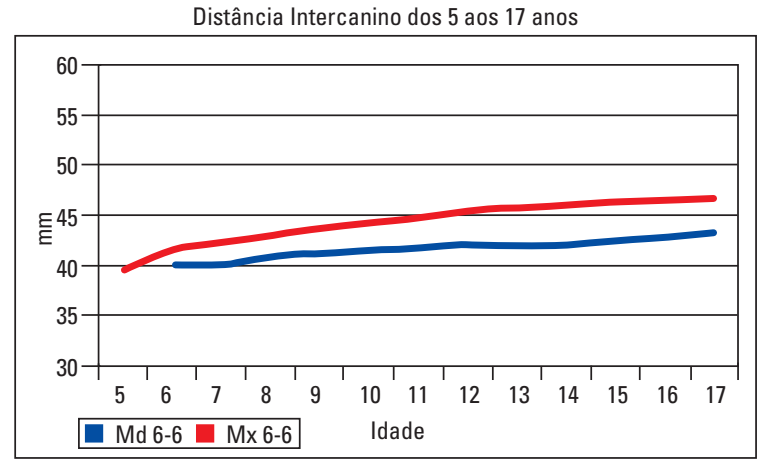

FIGURA 3 - Distância intercanino da maxila e da mandíbula dos 5 aos 17 anos de idade para o gênero masculino (MOYERS et al. ${ }^{21}, 1976$ ).

tou na maxila e mandíbula, com o maior valor encontrado na mandíbula para o gênero masculino, $0,33 \mathrm{~mm}$. Também houve redução do perímetro anterior dos arcos dentários maxilar e mandibular com valores em média de $-0,18 \mathrm{~mm}$ para maxila e $-0,38 \mathrm{~mm}$ na mandíbula. Os resultados permitiram aos autores concluir que os casos que se apresentam estáveis aos 23 anos de idade não passarão por grandes alterações nos próximos 10 anos.

\section{ARCO DENTÁRIO INFERIOR}

O desenvolvimento transversal do arco dentário inferior segue praticamente as mesmas características do arco superior, porém com valores e intensidades menores. Segundo o banco de dados de Moyers $^{21}$, a distância intercanino mandibular apresentou em média um aumento de 1,09 $\mathrm{mm}$ dos 3 aos 7 anos, aumento de 2,36 $\mathrm{mm}$ até os 12 anos e redução de $0,91 \mathrm{~mm}$ até os 18 anos. A distância intermolar aumentou 2,80 mm dos 6 aos 18 anos. Observe nas figuras 2 e 3 a comparação entre os arcos superior e inferior para as distâncias intercanino e intermolar avaliados longitudinalmente dos 5 aos 17 anos de idade. A distância intercanino mandibular apresenta uma aumento progressivo até os 9 e 10 anos de idade. A mesma correlação entre o desenvolvimento transversal dos arcos dentários e a erupção dentária encontrada na maxila foi observada na mandíbula, com uma diferença, após a irrupção dos incisivos laterais permanentes a distância intercanino praticamente se manteve até o final da dentição permanente (Fig. 4). Com relação 
Distância Intercanino Mandibular

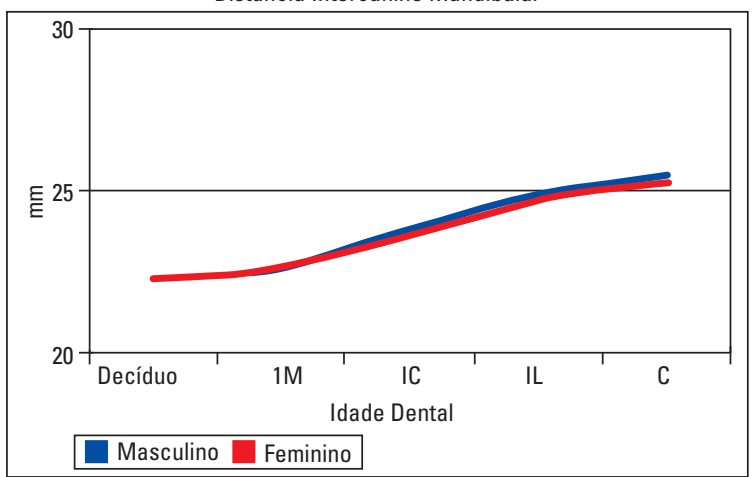

FIGURA 4 - Aumento da distância intercanino mandibular com relação à idade dental (irrupção dos dentes). Observe um aumento progressivo, o maior aumento ocorreu durante a troca dos incisivos central e lateral permanente e não durante a erupção dos caninos (MOORREES et al. $\left.{ }^{20}, 1964\right)(1 \mathrm{M}=$ primeiro molar permanente / IC= Incisivo central / IL=Incisivo lateral / $\mathrm{C}=$ Canino).

à distância intermolar, a mandíbula manteve uma curva ascendente até o final da adolescência.

Em média, o comprimento do arco dentário encontrado aos 18 anos de idade foi menor do que o observado aos 4 anos de idade, principalmente na mandíbula. Com relação à forma do arco, a mandíbula apresenta uma diminuição para ambos os gêneros, diferenciando-se da maxila que apresentou um leve aumento ${ }^{20}$. Na idade adulta, como na maxila, a dimensão transversal do arco dentário mandibular ou se manteve ou apresentou uma leve redução.

Agora que compreendemos um pouco mais sobre o crescimento normal dos arcos dentários focalizaremos exclusivamente as alterações normais das bases maxilares.

\section{CRESCIMENTO E DESENVOLVIMENTO TRANSVERSAL DOS MAXILARES}

Para facilitar a compreensão, o crescimento e desenvolvimento das bases ósseas foi dividido em duas partes, base maxilar e base mandibular.

\section{Base Maxilar}

Savara \& Singh ${ }^{24}$ acompanharam as modificações maxilares em meninos dos 3 aos 16 anos de idade. Os resultados demonstraram maiores modificações no crescimento para a dimensão vertical, ântero-posterior e por fim para a dimensão trans- versal. O padrão de modificação do gênero masculino foi similar ao padrão do gênero feminino, diferenciando somente no período do surto pubertário onde o crescimento maxilar ocorreu de 1 a 3 anos mais tarde para os meninos.

Melsen ${ }^{18}$ investigou o crescimento do palato duro num trabalho histológico em autópsia de material humano, realizado em pacientes que foram a óbito. $\mathrm{O}$ material foi obtido da remoção de blocos de $1 \mathrm{~cm}$ de tecido do palato duro da região posterior aos incisivos. A amostra foi composta por 33 pacientes do gênero masculino e 27 do gênero feminino, com idade de 0 a 18 anos. Nenhum paciente apresentou uma doença como causa do falecimento. Os resultados mostraram que a superfície nasal do palato duro reabsorveu até os 14, 15 anos de idade. Na superfície oral do palato foi detectada aposição óssea até a idade de 13, 14 anos para a maioria dos pacientes estudados. A morfologia das suturas mediana e transversa se alterou durante $\mathrm{o}$ crescimento pós-natal. A sutura mediana ao nascimento se encontrava larga, em formato de $\mathrm{Y}$ e com pouca sinuosidade. No período juvenil, a sinuosidade aumentou e no terceiro e último estágio a sutura possuía um curso tortuoso e com aumento da interdigitação. A autora concluiu que o maior responsável pelo crescimento vertical do palato duro foi o processo de remodelação óssea e não o abaixamento da parte anterior do palato. A grande descoberta desse trabalho foi a confirmação da oportunidade de crescimento transversal da sutura mediana após a adolescência. O crescimento transversal continuou até 16 anos para as meninas e 18 anos para os meninos.

Assim, ficou esclarecido qual era o período ativo do crescimento da sutura mediana, porém não estava determinado até que ponto o crescimento sutural era importante para o aumento transversal da maxila. Isso só foi possível determinar com a inserção de implantes metálicos na maxila em pacientes em fase de crescimento. Esse estudo foi preconizado por Björk e Skieller" ${ }^{4}$ que através do método de sobreposição de radiografias, com auxílio de implantes 


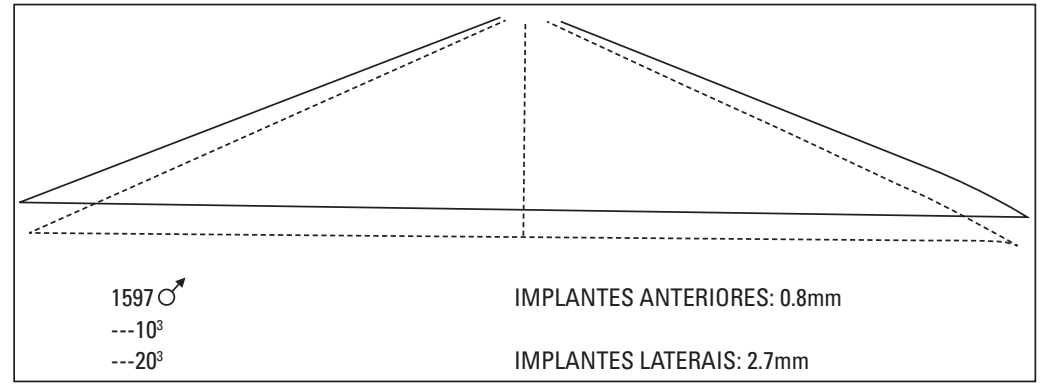

FIGURA 5 - Triângulo construído no plano transverso ilustrando a existência de uma rotação mútua entre as duas metades da maxila, note que o crescimento da sutura mediana foi maior para os implantes localizados na região posterior ( BJÖRK \& SKIELLER $\left.{ }^{4}, 1977\right)$. metálicos, analisaram o crescimento maxilar nas três dimensões. O início do acompanhamento foi aos 4 anos de idade e os pacientes selecionados apresentavam oclusão normal ou bem próxima do normal. O estudo revelou que o crescimento sutural inferior da maxila era duas vezes maior que a aposição óssea da parte inferior da órbita. A porção inferior da fossa nasal sofreu reabsorção enquanto a posição óssea foi observada no palato duro. O crescimento máximo puberal da sutura mediana coincidiu com o tempo de crescimento máximo das suturas faciais no plano sagital. O final do crescimento sutural ocorreu em média aos 17 anos de idade e simultaneamente para os planos sagital e transversal. $\mathrm{O}$ crescimento transverso entre os implantes posteriores foi em média $6,9 \mathrm{~mm}$ dos 4 anos de idade até a idade adulta (21 anos). O crescimento anual entre os implantes anteriores e posteriores foram em média de $0,12 \mathrm{~mm}$ e $0,42 \mathrm{~mm}$ respectivamente, com rotação transversal entre as duas metades da maxila. O contorno anterior do processo zigomático permaneceu estável e podendo este ser utilizado como estrutura de referência para estudos maxilares. Esse estudo veio a confirmar que o crescimento sutural é o fator mais importante para o desenvolvimento transversal da maxila e que a remodelação óssea também colabora com o desenvolvimento, porém, em proporções menores.

Um outro fator importante, relevado no trabalho de Björk e Skieller ${ }^{4}$, foi a presença de uma rotação no plano transverso da maxila, pois o cres- cimento da região posterior do palato foi maior que a região anterior (Fig. 5). Isto foi confirmado anos mais tarde por Korn \& Baumrind ${ }^{14}$ que estudaram o desenvolvimento transversal das bases esqueléticas da face em seres humanos dos 8 até os 15 anos de idade. $\mathrm{O}$ tamanho total da amostra foi de 31 pacientes, sendo 11 do gênero masculino e 20 do gênero feminino. A maxila e a mandíbula receberam implantes metálicos segundo a técnica descrita por Björk e Skieller ${ }^{4}$. As radiografias foram tomadas anualmente num período total de 9 anos. Os resultados demonstraram que o crescimento transversal na região posterior foi significantemente maior quando comparado à região anterior e mediana do palato, comprovando a existência de uma rotação maxilar no sentido transverso. As alterações anuais entre os implantes maxilares anteriores e posteriores foram de $0,15 \mathrm{e}$ $0,43 \mathrm{~mm}$ respectivamente. Quando considerado o gênero dos pacientes, o masculino apresentou valores superiores na região de incisivos e região zigomática. Na região anterior e posterior do palato o crescimento foi similar para ambos os gêneros. Portanto, não só ficou evidente a presença da rotação transversal da maxila como também a presença do crescimento transversal ativo após a infância.

Recentemente outros estudos confirmaram esse potencial de crescimento. Gandini \& Buschang ${ }^{8}$ avaliaram a estabilidade transversal dos maxilares. Participaram do estudo 25 pacientes, 12 do gênero masculino e 13 do gênero feminino com idade variando de 11.7 até 18.3 anos. Os pacientes apresentavam má oclusão de Classe I, apinhamento moderado e foram acompanhados por um período médio de 2.6 anos. Implantes metálicos foram inseridos na maxila e mandíbula também seguindo-se a técnica descrita por Björk e Skieller ${ }^{4}$ (Fig. 6). A amostra foi dividida de acordo com a intensidade de crescimento apresentada, assim dois subgrupos 


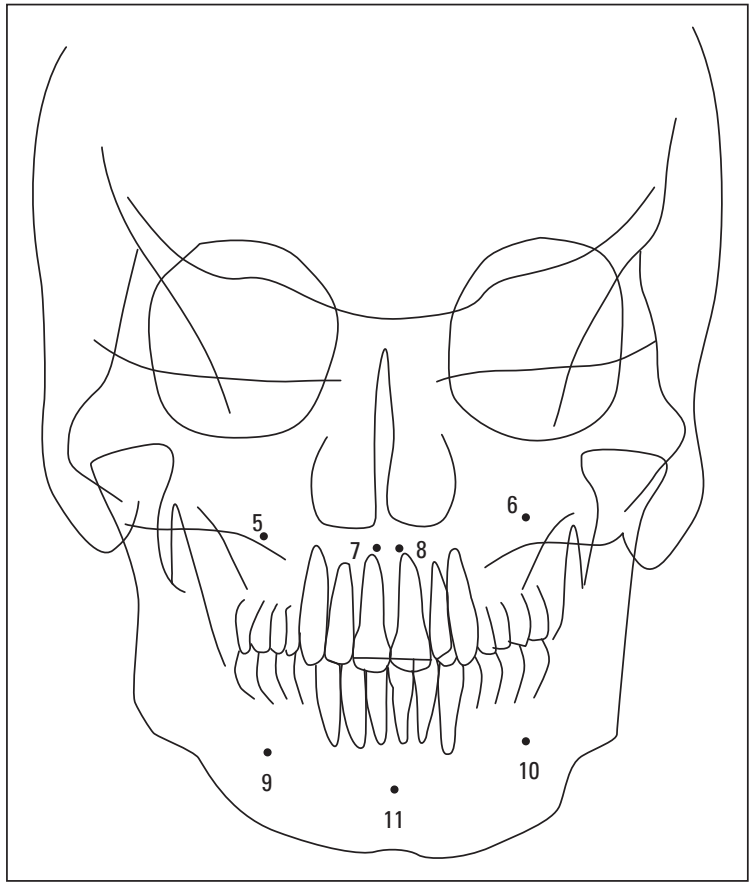

FIGURA 6 - Localização dos implantes metálicos inseridos na maxila e na mandíbula (GANDINI \& BUSCHANG ${ }^{8}, 2000$ ).

foram formados. O grupo de maior crescimento apresentou alterações transversais significativamente maiores que o grupo de menor crescimento. $\mathrm{O}$ aumento da distância entre os implantes maxilares foi de $0,8 \mathrm{~mm}$ (Fig. 7). A distância entre os implantes anteriores da maxila diminuiu $0,2 \mathrm{~mm}$, o que não foi significantemente diferente de zero. Contudo, foi observado um aumento anual de $0,27 \mathrm{~mm}$ entre os implantes maxilares posteriores, $-0,10 \mathrm{~mm}$ entre os implantes maxilares anteriores. Os autores concluíram que a dimensão transversal das estruturas basais da maxila e da mandíbula aumentou durante a adolescência. As alterações transversais foram maiores na maxila do que na mandíbula e estavam diretamente relacionadas ao potencial de crescimento individual.

O potencial de crescimento individual é outro fator considerável, pois a maioria dos trabalhos evidencia as médias acompanhadas do desvio padrão mostrando algumas vezes alta variabilidade. Pacientes com maior potencial de crescimento mostraram maiores alterações transversais e assim deve

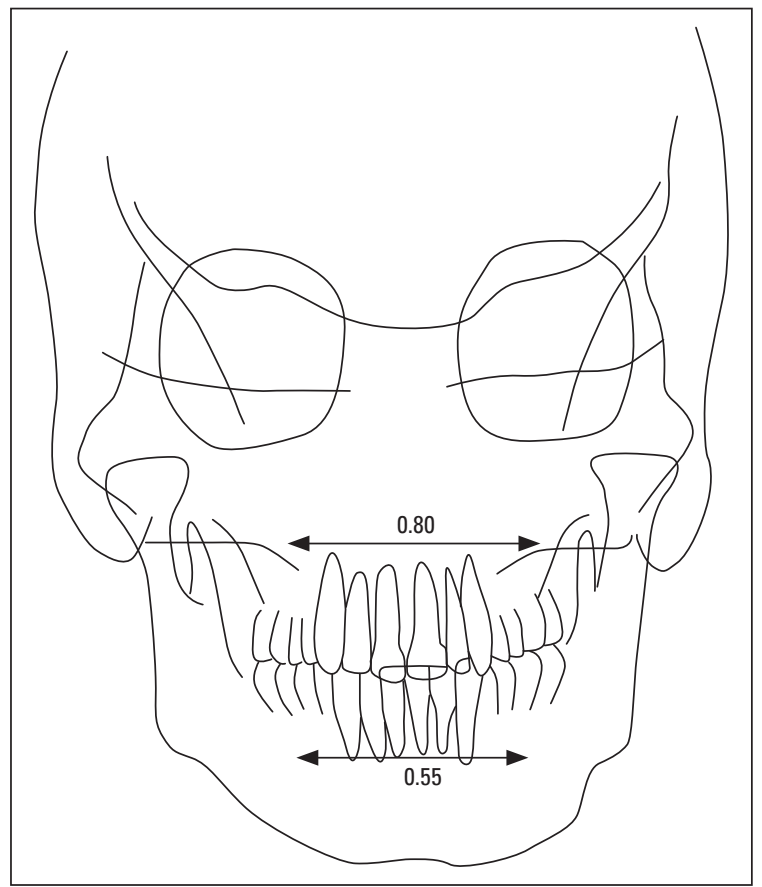

FIGURA 7 - Média do aumento $(\mathrm{mm})$ das distâncias entre os implante metálicos na maxila e na mandíbula durante o período de 2 anos e seis meses (GANDINI \& BUSCHANG ${ }^{8}, 2000$ ).

ser esperado maior tolerância quando relacionado à quantidade de expansão ortodôntica. Nota-se a importância dessas informações para o ortodontista pois, apesar do crescimento transversal maxilar existir até quase a idade adulta, o mesmo ocorre em diferentes locais, com diferentes magnitudes, e ainda está diretamente relacionado com o potencial de crescimento individual.

\section{Base Mandibular}

Tracy e Savara ${ }^{27}$ acompanharam as modificações mandibulares em meninas dos 3 aos 16 anos de idade. A amostra foi composta por 55 meninas que foram avaliadas anualmente. Durante o período entre 3 e 16 anos o comprimento do ramo mandibular aumentou $1,72 \mathrm{~cm}$. O comprimento mandibular, a dimensão que mais alterou, aumentou $3,09 \mathrm{~cm}$ e a distância bigonial e bicondilar aumentaram 2,05 $\mathrm{cm}$. O crescimento foi mais intenso no período circumpuberal principalmente para o comprimento mandibular, altura do ramo mandibular e distância bicondilar. 


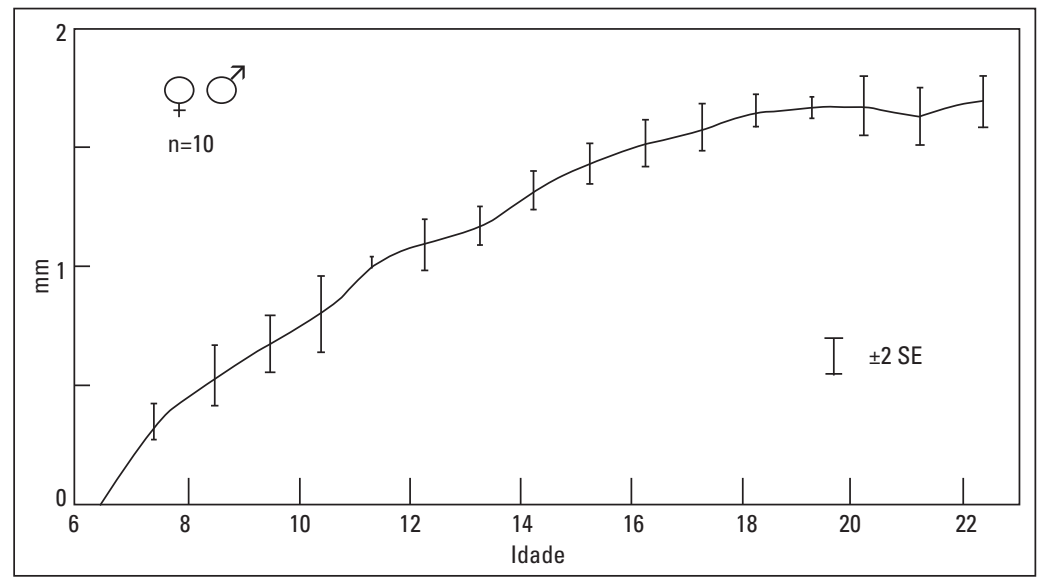

FIGURA 8 - Média em milímetros das alterações acumuladas da base mandibular mensurada entre os implantes metálicos inseridos na mandíbula. Note um aumento gradual na média da distância entre os implantes. Após os 18 anos, aumento praticamente permaneceu estável. As barras verticais representam o desvio padrão para cada ocasião (ISERI \& SOLOW'3, 2000).

cimento transversal da maxila foi relativamente maior que o da mandíbula, sendo um possivel mecanismo compensatório permitindo a preservação de uma oclusão posterior normal.

Iseri e Solow ${ }^{13}$ também mostraram existir um aumento progressivo da base mandibular. Os autores utilizaram uma amostra de 10 indivíduos com implantes metálicos bilaterais na mandíbula oriunda dos arquivos de Björk. O estudo foi composto por 3 pacientes do gênero feminino e 7 do gênero

Snodell et al. ${ }^{26}$, realizaram um estudo longitudinal cefalométrico do crescimento craniofacial. Os autores concluíram que o crescimento transversal da face apresenta intensidade menor e término mais precoce quando comparado às dimensões sagitais e verticais. Observaram ainda que o aumento transversal das bases ósseas foi maior para maxila do que para mandíbula e que após a erupção dos primeiros molares inferiores praticamente a dimensão transversal da mandíbula ficou estabilizada. Informações sobre os diferentes estágios do desenvolvimento transversal são de grande importância para o clínico já que as terapias ortodônticas que coincidem com o período de crescimento do paciente tendem a ser consideravelmente mais estáveis. Cortella et al. ${ }^{7}$, observaram que a velocidade do crescimento transversal da face coincidiu em média com o surto de crescimento pubertário, por volta de 11.5 anos para o gênero feminino e 13.5 anos para o gênero masculino. $O$ desenvolvimento transversal não cessou após o surto pubertário, a largura da mandíbula para o gênero masculino seguiu padrão similar ao desenvolvimento ântero-posterior e vertical. Foi observado que o crescimento em largura da mandíbula terminou antes que o crescimento sagital e vertical, com exceção da região posterior dos maxilares que continuou na mesma proporção que o crescimento sagital. O cres- masculino que foram observados dos 6 aos 23 anos de idade. Os resultados mostraram um aumento gradual entre os implantes mandibulares até os 18 anos de idade, após essa idade a distância praticamente se manteve inalterado até os 23 anos (Fig. 8). $\mathrm{O}$ aumento total observado no período entre 7 e 18 anos foi em média de 1,6 mm com desvio padrão de $0,42 \mathrm{~mm}$. Isto corresponde a um aumento anual de $0,13 \mathrm{~mm}$. A velocidade do aumento transversal diminuiu gradualmente dos 6 aos 10 anos, com dois posteriores picos com 11 e 14 anos e novamente uma redução até os 18 anos de idade. Os autores relataram as forças oclusais mastigatórias durante $\mathrm{o}$ crescimento pós-natal como uma possível explicação para esse aumento transversal da base mandibular. Essa carga mastigatória poderia influenciar a remodelação óssea do corpo mandibular produzindo ou permitindo um dobramento gradual permanente entre as duas metades mandibulares.

Em resumo, os estudos comprovam a existência de um aumento progressivo da dimensão transversal da maxila e da mandíbula. O período de maior intensidade mostrou ser coincidente com o surto de crescimento pubertário, após essa fase ainda é possivel encontrar discretas alterações, principalmente para o gênero masculino. As alterações parecem ser mais intensas para o gênero masculino, porém 


\begin{tabular}{|c|c|c|c|}
\hline \multicolumn{4}{|c|}{$\begin{array}{c}\text { Tabela } 1 \text { - Trabalhos relatando as alterações anuais (mm/ano) } \\
\text { entre implantes metálicos posicionados na maxila e na mandíbula. }\end{array}$} \\
\hline \multicolumn{4}{|c|}{ Alterações Anuais das Bases Maxilares } \\
\hline Pesquisas & $\begin{array}{l}\text { Distância entre os implantes } \\
\text { metálicos anteriores }\end{array}$ & $\begin{array}{l}\text { Distância entre os implantes } \\
\text { metálicos posteriores }\end{array}$ & $\begin{array}{l}\text { Distância entre os implantes } \\
\text { metálicos mandibulares }\end{array}$ \\
\hline Korn \& Baumrind ${ }^{14} n=31$ & $0.15(0.11)$ & $0.43(0.18)$ & $0.28 *(0.15)$ \\
\hline Gandini \& Buschang ${ }^{8} n=25$ & $-0.10(0.18)$ & $0.27(0.13)$ & $0.19(0.20)$ \\
\hline Björk \& Skiller ${ }^{4} n=09$ & $0.12(0.06)$ & $0.42(0.12)$ & - \\
\hline Iseri \& Solom ${ }^{13} n=10$ & - & - & $0.13^{* *}(0.06)$ \\
\hline
\end{tabular}

* Valor unilateral (0.14) 2. ** Valores mensurados até os 22 anos de idade

ocorrem mais tarde quando comparadas ao gênero feminino. Com relação ao paradigma de que a base mandibular não se altera transversalmente após a fusão da sutura sinfiseana parece não ser verdadeiro. Estudos confirmam um aumento transversal das estruturas basais da mandíbula. Korn e Baumrind ${ }^{14}$ mostraram que a distância entre os implantes posteriores na mandíbula aumentou em média 0,28 $\mathrm{mm}$ por ano, Gandini e Buschang ${ }^{8}$ mostraram existir um aumento entre os implantes mandibulares de 0,19 mm num período de 2,6 anos, a amostra utilizada apresentava idade mais elevada e conseqüentemente menor potencial de crescimento. Iseri $\&$ Solow ${ }^{13}$ relataram um aumento entre 7 e 18 anos de idade de 1,6 mm em média com desvio padrão de $0,42 \mathrm{~mm}$. Isto corresponde a um aumento anual de 0,13 mm (Tab. 1). Essa média foi inferior ao valor encontrado por Korn e Baumrind ${ }^{14}$, de 0,28 mm por ano, porém no trabalho de Iseri \& Solow ${ }^{13}$ o período avaliado foi até os 22 anos de idade, sendo que após os 18 anos o aumento observado foi praticamente zero e até negativo, conseqüentemente o valor da média anual foi diminuído. Desta forma, podemos esperar por um aumento da base mandibular num período, por exemplo, dos 6 aos 16 nos de até $3 \mathrm{~mm}$, dependendo do potencial individual de crescimento esse valor pode ser ainda maior ou obviamente menor.

A origem do aumento transversal da mandíbula ainda não está totalmente definida, alguns autores acreditam numa acomodação na região da sínfise mandibular, outros relataram que as forças oclusais mastigatórias produzem um dobramento gradual permanente entre as duas metades mandibulares. Novos trabalhos na área biomecânica das forças mastigatórias devem ser realizados para total compreensão dos efeitos associados.

\section{COMPARAÇÃO ENTRE O DESENVOLVIMENTO DOS ARCOS DENTÁRIOS COM AS BASES MAXILARES}

Observe a comparação apresentada nas figuras 9 e 10. Dos 6 aos 12 anos, as distâncias intercanino e intermolar no arco superior aumentaram respectivamente 5,01 e 3,49 $\mathrm{mm}$. Observe que durante o mesmo período a base maxilar anterior e posterior também aumentou, sendo esse aumento mais evidente para região posterior da maxila, 2,52 mm entre os implantes metálicos localizados no processo zigomático. Na mandíbula foi observado semelhante resultado com aumento de $2,81 \mathrm{~mm}$ para a distância intercanino, 1,93 $\mathrm{mm}$ para a distância intermolar e 1,7 mm entre os implantes localizados na região de primeiros molares inferiores. Desta forma, observamos que o arco dentário de uma maneira geral apresenta um aumento transversal superior à sua base óssea até os 12 anos de idade.

Após os 12 anos para ser mais preciso até os 17 anos de idade, a distância intercanino superior e inferior diminuíram em aproximadamente $0,5 \mathrm{~mm}$ e a distância intermolar superior como a inferior aumentaram, 1,72 e 1,28 mm respectivamente. As bases ósseas continuaram a crescer transversalmente porém desta vez apresentaram valores superiores 


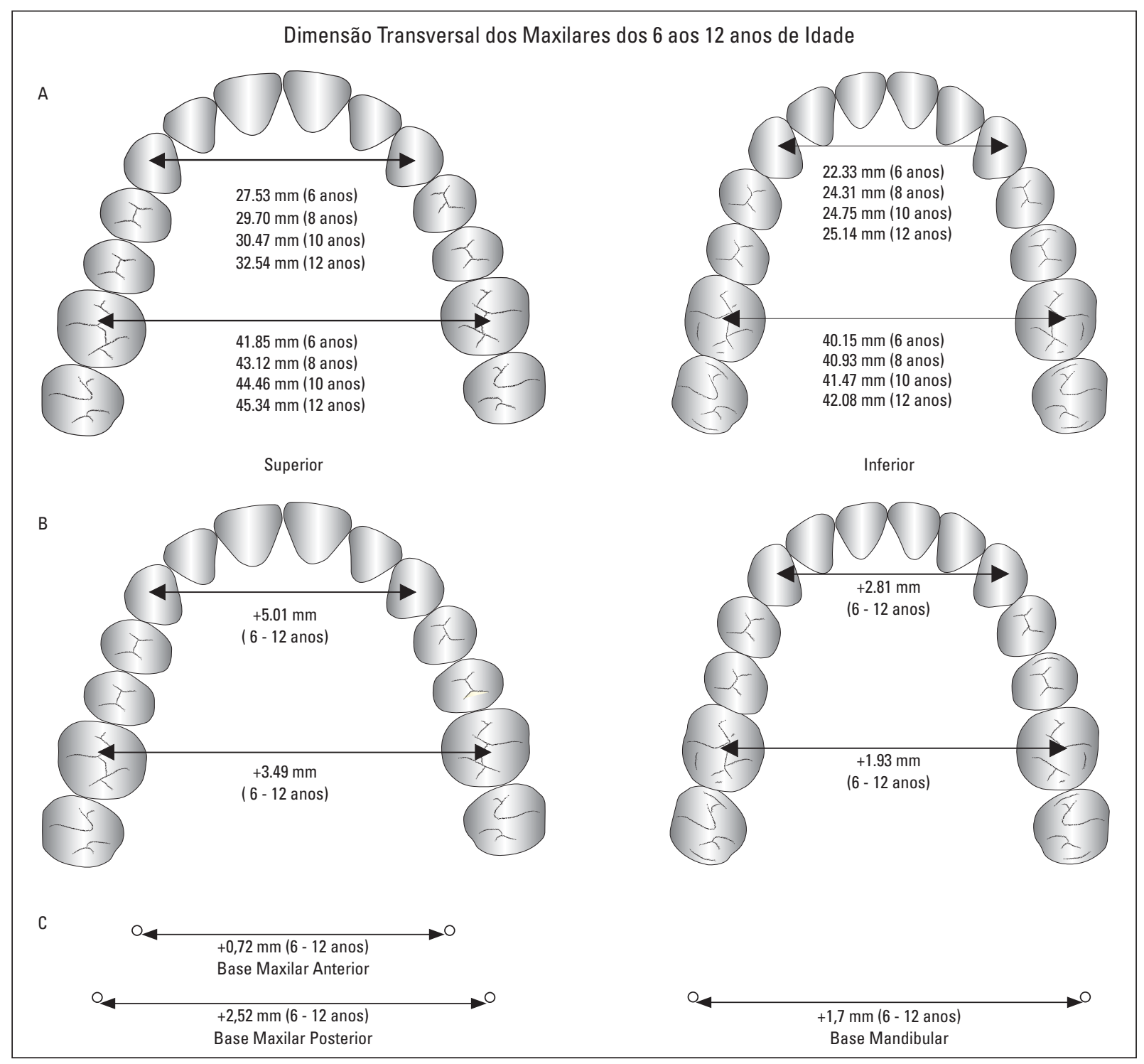

FIGURA 9 - Dimensão transversal dos maxilares dos 6 aos 12 anos de idade do gênero masculino, $\mathbf{A}$ - Alterações presentes nas distâncias intercanino e intermolar para ambos os arcos, B - Total das alterações presente nos arcos dentários dos 6 aos 12 anos, C - Total de crescimento da base óssea maxilar e mandibular no mesmo período mensurado entre implantes metálicos. Na maxila os implantes posteriores foram inseridos no processo zigomático na direção dos primeiros molares e os anteriores na região da espinha nasal anterior. Na mandíbula os implantes foram posicionados inferiormente aos primeiros molares (MOYERS et al. ${ }^{21}, 1976$ / BJÖRK \& SKIELLER ${ }^{4}, 1977$ / KORN \& BAUMRIND $\left.{ }^{14}, 1990\right)$.

quando comparados com a expansão dento-alveolar (Fig. 10). Agora se as bases ósseas continuam a crescer transversalmente até a idade adulta por que não encontramos a mesma proporção para o arco dentário, ou melhor, por que as distâncias intercanino em vez de aumentarem, elas diminuíram? Uma possível explicação é a migração mesial dos dentes associada à diminuição do comprimento do arco dentário que ocorre nessa fase. Se lembrarmos que os dentes estão posicionados em um arco, quanto mais anterior eles estiverem menores serão as distâncias transversais. Alguns fatores como os espaços primatas, o "Leeway space" e até um possível apinhamento colaboram com essa migração mesial dos dentes e conseqüente diminuição da dimensão transversal dos arcos dentários. 


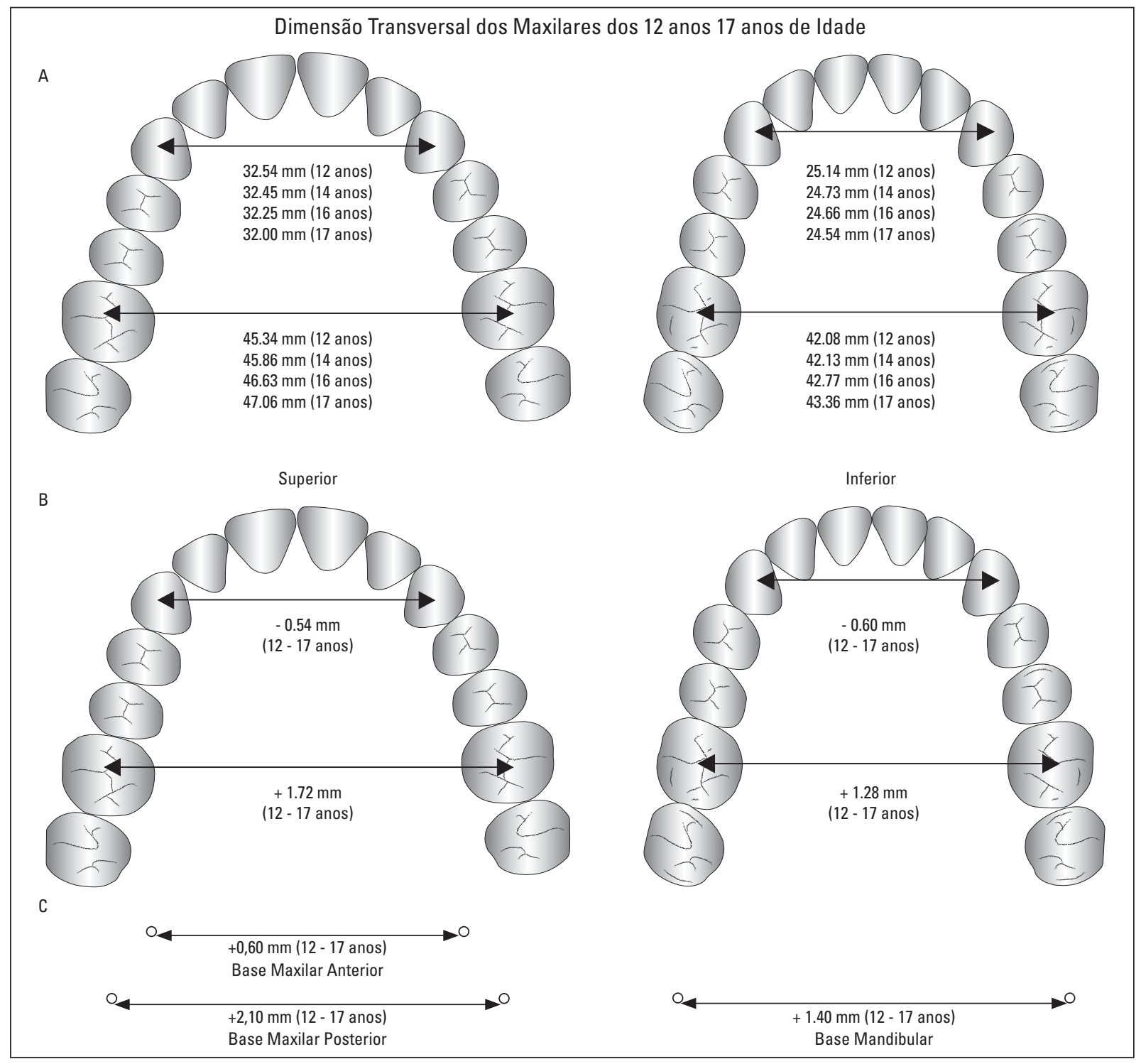

FIGURA 10 - Dimensão transversal dos maxilares dos 12 aos 17 anos de idade para o gênero masculino, $\mathbf{A}$ - Alterações presentes nas distâncias intercanino e intermolar para ambos os arcos, B - Total das alterações presente nos arcos dentários dos 12 aos 17 anos, C - Total de crescimento da base óssea maxilar e mandibular no mesmo período mensurado entre implantes metálicos. Na maxila os implantes posteriores foram inseridos no processo zigomático na direção dos primeiros molares e os anteriores na região da espinha nasal anterior. Na mandíbula os implantes foram posicionados inferiormente aos primeiros molares (MOYERS et al. ${ }^{21}$, 1976 / BJÖRK \& SKIELLER ${ }^{4}, 1977$ / KORN \& BAUMRIND $\left.{ }^{14}, 1990\right)$.

Indivíduos com maior aumento na região posterior da maxila também mostraram maior aumento da base mandibular entre os implantes mandibulares $^{8}$. Clinicamente, uma vez expandido o arco dentário superior o arco inferior tende a aumentar conseqüentemente sem intervenção ortodôntica ou mesmo se tratado ortodonticamente os resultados parecem ser mais estáveis ${ }^{23}$. Da mesma manei- ra que a maxila, a região posterior da mandíbula mostrou um crescimento maior que a região anterior com o centro de rotação transversal localizado possivelmente na região sinfiseana. Portanto, o aumento transversal maxilar e mandibular aqui discutido é real, porém em pequenas magnitudes e quanto maior for a idade do paciente menor será a possibilidade de alteração da dimensão transversal. 


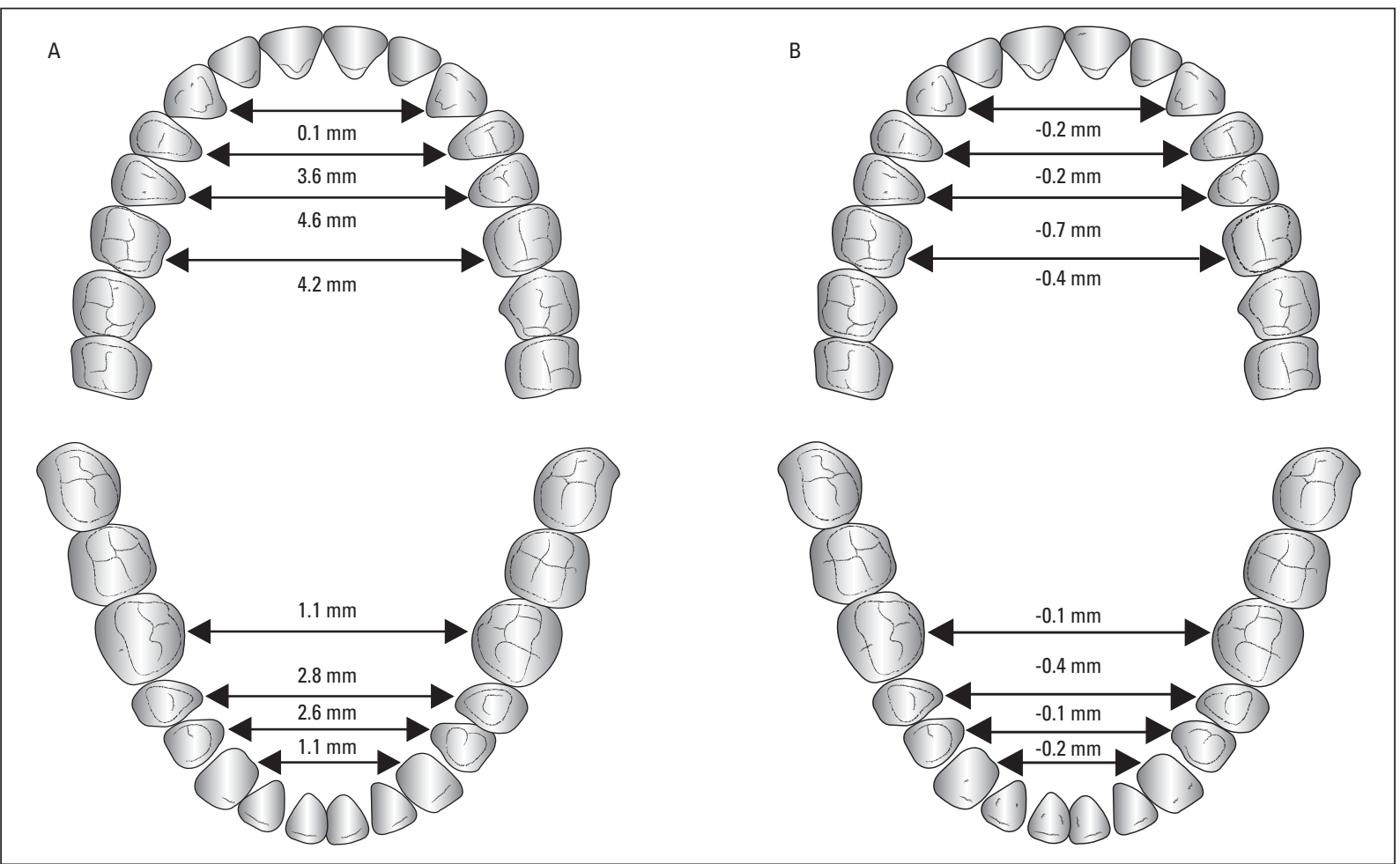

FIGURA 11 - Alterações durante o tratamento A) e no pós-tratamento B) mensuradas em milímetros no limite gengival dos dentes para o grupo de pacientes que usaram contenção. (BUSCHANG et al. ${ }^{6}, 2001$ ).

Clinicamente, a seguir a questão que deve ser discutida principalmente para o arco inferior: o que acontece se impedirmos esse movimento mesial dos dentes e usufruirmos o espaço remanescente? $\mathrm{Ou}$ ainda, se em determinada fase do desenvolvimento, as bases ósseas crescem mais que os arcos dentários, por que não expandir os arcos dentários?

\section{APLICAÇÃO CLÍNICA DA EXPANSÃO MANDIBULAR}

Autores como Herberger e outros, Little e ou$\operatorname{tros}^{11,16,17}$ acreditam que a distância intercanino na mandíbula deve ser inviolável, partindo do princípio que a dimensão anterior do arco dentário é precocemente estabelecida e mantida por várias décadas devido ao equilíbrio muscular presente. Uma alteração da distância intercanino, como uma expansão na região anterior do arco, poderá causar um desequilíbrio no sistema levando a uma possível instabilidade dos resultados. Essa é uma teo- ria clássica que se perpetua por muitos anos e na literatura existem trabalhos que comprovam essa estabilidade.

Ao mesmo tempo, sabemos que alguns trabalhos mostram que alterações transversais no arco mandibular com aparelhos ortodônticos são possíveis e relativamente estáveis ${ }^{6,12,15}$. Buschang et al. ${ }^{6}$ fizeram uma revisão de três trabalhos avaliando diferentes maneiras para correção da discrepância no comprimento do arco dentário segundo a disciplina de Alexander. O primeiro trabalho avaliou os efeitos da terapia com diferentes placas lábio-ativas (PLA) sobre o arco dentário. Os resultados mostraram que a PLA com escudo de acrílico na região anterior produziu maior deslocamento da musculatura labial e conseqüentemente maior deslocamento distal dos molares inferiores. Independente do tipo de aparelho e da conduta clínica as coroas dos incisivos inferiores inclinaram para vestibular enquanto os ápices se mantiveram inalterados e o simples fato 


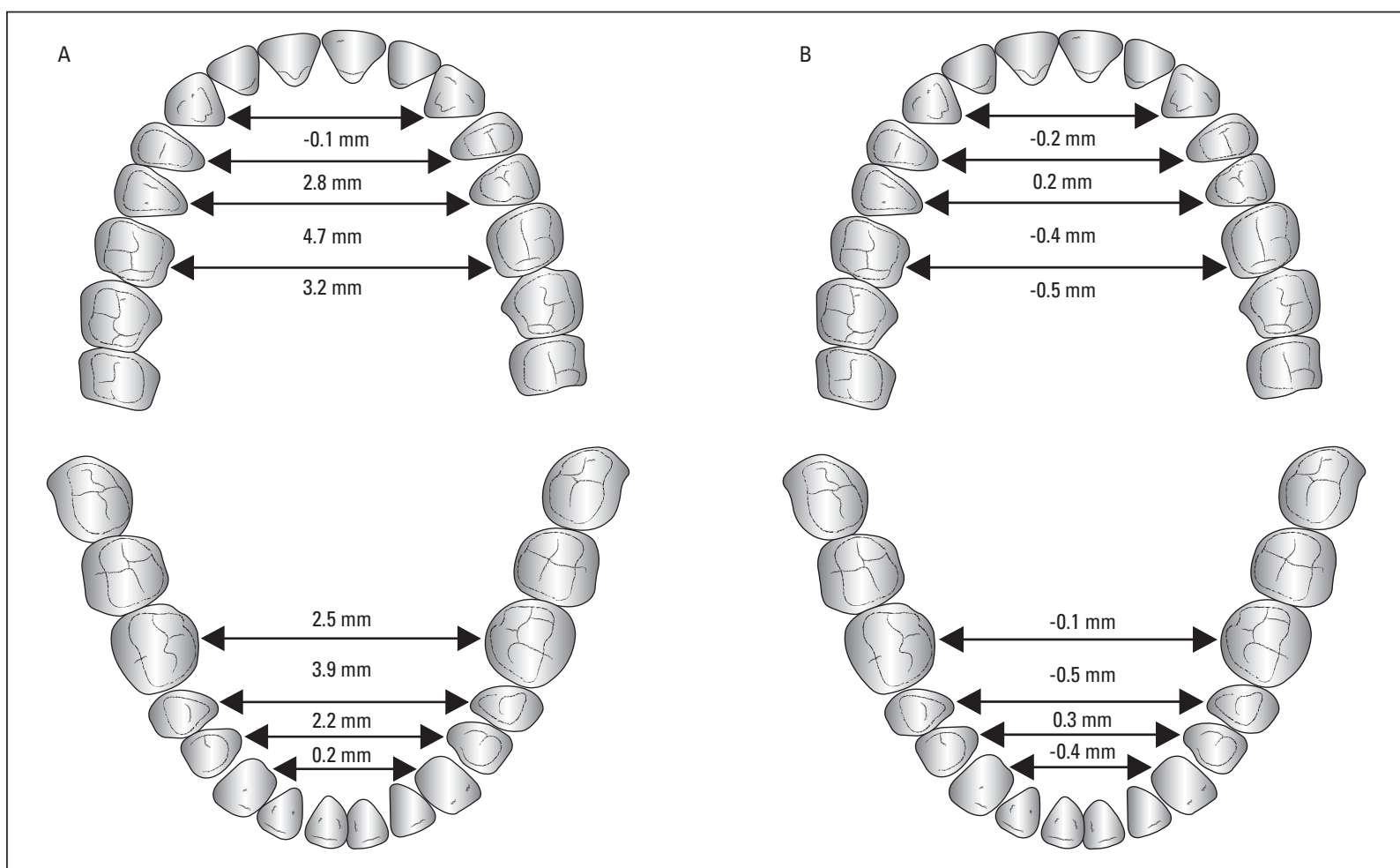

FIGURA 12 - Alterações durante o tratamento $\mathbf{A}$ ) e no pós-tratamento $\mathbf{B}$ ) mensuradas em milímetros no limite gengival dos dentes para o grupo de pacientes que não usaram contenção. (BUSCHANG et al. $\left.{ }^{6}, 2001\right)$

de remover a pressão da musculatura labial também causou similar efeito sobre os caninos. O segundo trabalho apresentado tinha o objetivo de avaliar os efeitos de diferentes expansores removíveis sobre o arco dentário inferior. Os aparelhos PLA e expansor removivel (ER) foram comparados. Os autores concluíram que a PLA é indicada para más oclusões onde os incisivos estejam verticalizados e os molares inferiores com inclinação mesial pois inclinação vestibular dos incisivos, extrusão e inclinação distal dos molares são esperados. A PLA foi idealmente preconizada para casos com apinhamentos localizados na região anterior do arco inferior e para preservação do "leeway space" após esfoliação dos molares decíduos. Quando comparado ao aparelho ER a PLA mostrou um aumento superior para a largura posterior do arco dentário mandibular. O ER foi idealmente preconizado para más oclusões com apinhamentos moderados na região anterior do arco dentário e que os incisivos não necessariamente precisam estar ver- ticalizados já que o espaço criado para o alinhamento dos incisivos é oriundo do aumento da distância intercanino e não da vestibularização dos incisivos. $O$ ER pode ser utilizado em pacientes com tendência de mordida aberta já que a porção posterior de acrílico do aparelho limita a erupção dos segmentos posteriores e permite uma erupção passiva dos incisivos. O ER não deve ser indicado para correção de apinhamento posterior ou manutenção do "leeway space". O terceiro e mais importante trabalho discutido avaliou a estabilidade transversal dos arcos dentários quando submetidos à terapia simultânea de expansão rápida da maxila e placa lábio-ativa com conseqüente finalização com a terapia ortodôntica corretiva. A amostra foi subdividida em dois grupos de acordo com a contenção utilizada. Um grupo usou retentor inferior tipo barra 3-3 enquanto o outro grupo não utilizou nenhum tipo de contenção e os resultados foram avaliados 1 ano após a remoção do aparelho fixo. O tratamento aumentou a dimen- 
são transversal dos arcos dentários. Com exceção para a profundidade dos arcos dentários, o grupo de contenção se mostrou estável no período pós-tratamento, mas para o grupo sem contenção algumas alterações ocorreram. A distância intercanino inferior recidivou $50 \%$ do total expandido, porém na região posterior a recidiva foi aproximadamente de $10 \%$ do total expandido. Para ambos os arcos os resultados foram considerados estáveis após um ano de pós-tratamento já que estudos confirmam que as maiores recidivas ocorrem num curto período após o término do tratamento. Observe nas figuras 11 e 12 o total de expansão obtido e a estabilidade dos resultados.

A dimensão transversal mandibular já considerada inalterável pode ser definitivamente aumentada em pacientes jovens que inicialmente apresentam constrição no arco inferior e superior. Os expansores inferiores mostraram sua efetividade e, quando associados a uma rápida expansão da maxila e tratamento ortodôntico corretivo, melhor resultado pode ser esperado, já que melhores inclinações axiais podem ser obtidas e estabilizadas. Esse é um fator importante que deve ser ressaltado, pois nos trabalhos cujo arco mandibular foi expandido e relatado uma satisfatória estabilidade, a expansão maior ocorreu na região posterior e foram selecionados pacientes com atresia do arco superior e inferior e os mesmos se encontravam na dentição mista, período de maior desenvolvimento da dimensão transversal do arco inferior. A grande incógnita que ainda persiste é qual seria a estabilidade dos resultados caso a expansão do arco mandibular fosse preconizada em pacientes com dimensão transversal normal do arco superior e/ou inferior e apinhamento dentário ou ainda se realizado em pacientes adultos.

\section{CONCLUSÃO}

O paradigma instituído no início do século passado e perpetuado até hoje por alguns seguidores de que a dimensão transversal dos arcos dentários não pode ser alterada não faz sentido nos dias atuais. A literatura nos mostrou que as bases maxilares continuam a se desenvolver transversalmente até a idade adulta. Mesmo a mandíbula após a fusão da sutura sinfiseana também mostrou potencial de crescimento transversal. Os arcos dentários aumentam progressivamente no sentido transversal até a dentição permanente e a oportunidade de expansão da distância intercanino e intermolar pode ser considerada. Não podemos concluir que uma grande expansão na região anterior da mandíbula seja estável, pois a literatura não apresenta provas que confirmem esta definição, porém alteração na dimensão transversal do arco dentário mandibular parece ser possível e relativamente estável quando realizada em arcos dentários atrésicos e em paciente jovem. Devemos idealmente diminuir a atenção sobre aparatologia e sim compreender como e quando as estruturas faciais se desenvolvem e como as mesmas são influenciadas pelas más oclusões, assim teremos condições de oferecer aos nossos pacientes alternativas capazes de proporcionar um crescimento transversal normal dos maxilares.

\section{AGRADECIMENTO}

CAPES - Bolsista Doutorado Sanduíche e ao Prof. Dr. Luiz Gandini Jr e Prof. Dr. Marcelo M. Araújo pela revisão científica do trabalho.

Enviado em: Dezembro de 2002 Revisado e aceito: Abril de 2003

\section{Transversal growth and development of the jaws - News opportunities for mandibular expansion}

\footnotetext{
Abstract

The growth and adaptation of the craniofacial complex has been a longstanding interest of orthodontists who understand that normal growth changes provide an essential reference for diagnosis and treatment planning.
} 
With recent developments there has been increased research activity pertaining to transverse developmental changes on both treated and untreated subjects. Of particular importance, implants studies evaluating normal developmental changes of the skeletal bases have forced orthodontists to reconsider the long notions established from longitudinal descriptions of the dental arches changes. These more recent studies defy the notion that transverse mandibular arch dimensions are immutable. The purpose of this paper is to 1) synthetize the literature pertaining to transverse maxillary and mandibular arch changes of untreated children and adolescents and 2) discuss possible clinical implications of these transverse arch changes during growth.

Key words: Craniofacial growth. Maxillary expansion. Mandibular expansion.

\section{REFERÊNCIAS}

1. ATHANASIOU, A. E.; DERM, H. D.: BOSCH, C. Data and patterns of transverse dentofacial structure of 6- to 15-yearsold children: A posterior cephalometric study. Am J Orthod Dentofacial Orthop, St. Louis, v.102, p. 465-471, 1992.

2. BISHARA, S. E.; JAKOBSEN, J. R.; TREDER, J. T.; NOWAK, A. Arch width changes from 6 weeks to 45 of age. Am J Orthod Dentofacial Orthop, St. Louis, v. 111, p. 401-409, 1997.

3. BISHARA, S.E.; JAKOBSEN, J. R.; TREDER, J. T.; STASI, M.J. Changes in the maxillary and mandibular tooth size-arch length relationship from early adolescence to early adulthood. Am J Orthod Dentofacial Orthop, St. Louis, v. 95, p. 46-59, 1989.

4. BJÖRK, A.; SKIELLER, V. Growth of the maxilla in three dimensions as revealed radiographically by the implant metallic method. Br J Orthod, London, v. 4. p. 53-64,1977.

5. BONDEVIK, O. Changes in occlusion between 23 and 34 years. Angle Orthod, Appleton, v. 68, p. 75-80, 1998.

6. BUSCHANG, P. H.; HORTON-REULAND, S. J.; NEVANT, C. Nonextraction approach to tooth size arch length discrepancies with the Alexander discipline. Semin Orthod, Orlando, v. 7, p.117-131, 2001

7. CORTELLA, S.; SHOFER, F. S.; GHAFARI, J. Transverse development of the jaws: norms for the posterioranterior cephalometric analysis. Am J Orthod Dentofacial Orthop, St. Louis, v. 112 , p. 519-522, 1997

8. GANDINI Jr., L. G.; BUSCHANG, P. H. Maxillary and mandibular width changes studied using metallic implantes. Am J Orthod Dentofacial Orthop, St. Louis, v. 117, p. 75-80, 2000.

9. GRABER, T. M.; VANARSDALL, Jr., R. L. Orthodontics: current principles and techniques. 2nd ed. St. Louis: C. V. Mosby, 1994. p. 965.

10. HARRIS, E. F. A longitudinal study of arch of arch size and form in untreated adults. Am J Orthod Dentofacial Orthop, St. Louis, v. 111, p. 419-427, 1997.

11. HERBERGER, R. J. Stability of mandibular intercuspid width after long periods of retention. Angle Orthod, Appleton, v. 51, p. 613-617, 1981.

12. HORTON, S. J. The transverse stability of combined rapid palatal expansion and lip bumper therapy following comprehensive orthodontic treatment. 1997. Master's (Thesis)submitted to Baylor University, Dallas, Texas, 1997.

13. ISERI, H.; SOLOW, B. Change in the width of the mandibular body from 6 to 23 years of age: an implant study. Eur J Orthod, London, v. 22, p. 229, 2000

14. KORN, E. L.; BAUMRIND, S. Transverse development of the human jaws between the ages of 8.5 and 15.5 years, studied longitudinally with use of implants. J Dent Res, Chicago, v. 69, p.1298-1306, 1990.

15. LEGRER, L. R. The effects of removable expansion on the mandible arch. 1991. Master's (Thesis) -submitted to Baylor University, Dallas, Texas, 1991.
16. LITTLE, R. M.; RIEDEL, R. A.; ARTUN, J. An evaluation of changes in mandibular anterior alignment from 10 to 20 years postretention. Am J Orthod Dentofacial Orthop, St. Louis, v. 93, p. 423-428, 1988

17. LITTLE, R. M.; WALLEN, T. R.; RIEDEL, R. A. Stability and relapse of mandibular anterior alignment first premolar extraction cases treated by traditional edgewise orthodontics. Am J Orthod Dentofacial Orthop, St. Louis, v. 80, p. 349-365, 1981

18. MELSEN, B. Palatal growth studied on human autopsy material. Am J Orthod Dentofacial Orthop, St. Louis, v. 68, p. 42-54 1975.

19. MOORREES, C.F. A.; GRON, A.; LEBRET, L. M. L.; YEN, P. K. J.; FROHLICH, F. J. Growth studies of the dentition: A review. Am J Orthod Dentofacial Orthop, St. Louis, v. 55, p. 601-617, 1969.

20. MOORREES, C. F. A.; REED, R. B. Changes in dental arch dimensions expressed on the basis of tooth eruption as a measure of biologic age. J Dental Res, Chicago, v. 44, p. 129-141, 1964.

21. MOYERS, R.; LINDEN, F. van der; RIOLO, M. Standards of human occlusal development. In: CARLSON, D. S. Craniofacial biology. Ann Arbor: [s.n.], 1976. p. 371. Craniofacial Growth Series.

22. RICHARDSON, M. E. Late lower arch crowding: The role of the transverse dimension. Am J Orthod Dentofacial Orthop, St. Louis, v.107, p. 613-617, 1995

23. SANDSTROM, R. A.; KLAPPEER, L.; PAPACONSTANTINOU, $\mathrm{S}$. Expansion of the lower arch concurrent with rapid maxillary expansion. Am J Orthod Dentofacial Orthop, St. Louis, v. 94 p. 296-302, 1988

24. SAVARA, B. S.; SINGH, I. J. Norms of size and annual increments of seven anatomical measures of maxillae in boys from three to sixteen years of age. Angle Orthod, Appleton, v. 38 p.104-120, 1968

25. SINCLAIR, P. M.; LITTLE, R. M. Maturation of untreated norma occlusions. Am J Orthod Dentofacial Orthop, St. Louis, v. 83 p.114-123, 1983.

26. SNODELL, S. F.; NANDA, R. S.; CURRIER, G. F. A longitudinal cephalometric study of transverse and vertical craniofacial growth. Am J Orthod Dentofacial Orthop, St. Louis, v. 104, p. 471-483, 1993.

27. TRACY, W. E.; SAVARA, B. S. Norms of size and annual increments of five anatomical measures of the mandible in girls from 3 to 16 years of age. Archs O Bio, [S. I.], v. 11, p. 587-598, 1966.

\section{Endereço para correspondência}

Adriano Marotta Araujo

Rua Marcondes Salgado, 64

CEP: 12243-820 - São José dos Campos - SP, Brasil

dradriano@orthoi.com.br 